\title{
Deutschunterricht in Firmenkursen I. Ergebnisse einer Umfrage aus dem Sommer 2009
}

\section{Pavla Marečková}

In the last few years, the number of companies and institutions that organize a language course for their employees has been growing rapidly. From the viewpoint of methodology, however, there is a lack of technical literature discussing the regularities of such language courses. That is why a survey was conducted with teachers and participants of these courses that was meant to outline the present situation. To begin with, the paper at hand presents the evaluation of the teachers' answers. Further results including the students' responses will be presented, due to space restrictions, in the next issue of this magazine.

company language courses - German as a foreign language - foreign language teaching language polls - questionnaire

Die Zahl der Firmen bzw. Institutionen, die für ihre Angestellten einen Sprachkurs organisieren, nimmt in den letzten Jahren stark zu. Aus didaktischer Sicht fehlt es jedoch an Fachliteratur, die die Gesetzmäßigkeiten eines solchen Fremdsprachenunterrichts komplex behandeln würde. Daher wurde eine Umfrage unter LektorInnen und KursteilnehmerInnen durchgeführt, die eine Vorstellung über die gegenwärtige Situation ermöglichen sollte. Im vorliegenden Beitrag werden zunächst die Ergebnisse präsentiert, die die Antworten der LektorInnen widerspiegeln. Die weiteren Ergebnisse erscheinen aus Platzgründen in der nächsten Nummer dieser Zeitschrift.

Firmenunterricht - Firmenkurs - Umfrage - Deutsch als Fremdsprache Fremdsprachendidaktik

\section{Einleitung}

Das Thema des Fremdsprachenlernens und -lehrens gehört seit mehreren Jahren nicht nur in die Schulen, sondern wird immer häufiger im Zusammenhang mit der Berufswelt diskutiert. Attraktive Arbeitschancen und neue Handelsmöglichkeiten innerhalb sowie außerhalb Europas haben zur Folge, dass berufsorientierte Sprachkurse für Erwachsene sich eines steigenden Interesses erfreuen. Dieses Interesse an lebenslangem Fremdsprachenlernen für den Beruf wird auch von der Sprachenpolitik der Europäischen Union unterstützt. Im Gemeinsamen europäischen Referenzrahmen für Sprachen werden folgende Handlungsziele auf dem Gebiet der modernen Sprachen formuliert:

- „alle Europäerinnen und Europäer mit dem nötigen Rüstzeug auszustatten für die Herausforderungen verstärkter internationaler Mobilität und engerer Zusammenarbeit, nicht nur im Bildungswesen und in kulturellen und wissenschaftlichen Belangen, sondern auch in Handel und Industrie; 
- den Bedürfnissen eines vielsprachigen und multikulturellen Europas dadurch entgegenzukommen, dass die Fähigkeit der Europäer, über sprachliche und kulturelle Grenzen hinweg miteinander zu kommunizieren, deutlich verbessert wird. Das erfordert von ihnen ununterbrochene, lebenslange Bemühungen, zu denen sie ermutigt werden müssen; diese Bemühungen müssen auf organisatorisch sicherem Boden stehen und auf allen Ebenen des Bildungswesens von den zuständigen Körperschaften finanziert werden.“ (GERR: 2000)

Obwohl die deutsche Sprache heutzutage nicht gerade zu den heißen Trends an Grund- bzw. Hauptschulen zählt, sieht die Situation im Berufsbereich gewissermaßen anders aus. Nach NEKULA $(2004,129)$ wird das Deutsche durch die geographische Lage Tschechiens sowie durch die starke Präsenz des deutschen, österreichischen und niederländischen Kapitals in Tschechien in diesem Bereich im Vergleich mit dem Englischen etwa gleich nachgefragt. Von dieser Seite dürften auch neue Impulse für das tschechische Bildungssystem ausgehen, auch wenn es keine Ausnahme ist, dass deutsche bzw. deutsch-tschechische Unternehmen oder internationale Konzerne mit deutscher Beteiligung von ihren tschechischen Mitarbeitern statt der deutschen Sprache englische Sprachkompetenz verlangen. Hier sind aber durchaus regionale Unterschiede festzustellen, eine Rolle spielt auch die Größe und Ausrichtung des Unternehmens.

\section{Spezifika von Firmenkursen}

Um den oben genannten Zielen der Sprachenpolitik der EU sowie der Nachfrage seitens der Arbeitgeber sowie der Angestellten nachzukommen, ist es erforderlich ein neues Kapitel der Fremdsprachendidaktik zu schreiben, in dem die Gesetzmäßigkeiten und Spezifika des Fremdsprachenunterrichts in Firmenkursen bzw. in anderen berufsorientierten Sprachkursen, beschrieben würden. Diese Kurse unterscheiden sich nämlich von anderen Sprachkursen durch mehrere Merkmale, seien es die sprachlichen Ziele und Inhalte, die oft von dem jeweiligen Arbeitsfeld abhängig sind, oder die Methodik, die der Zielgruppe angepasst werden muss.

\section{Forschungsvorhaben und -vorgehen}

In diesem Beitrag konzentrieren wir uns auf den Deutschunterricht in Firmenkursen, d. h. in Sprachkursen, die von Arbeitgebern für die Arbeitnehmer organisiert werden (z. B. mittels einer Sprachschule) und die direkt in der Firma oder in der Sprachschule stattfinden. Mit dem Ziel die wesentlichen Aspekte dieses Unterrichts festzustellen und zu beschreiben, haben wir eine Untersuchung durchgeführt, deren Ergebnisse eine Einsicht in die Problematik ermöglichen sollen. Die Umfrage wurde 
im Rahmen des Forschungsprojektes der Masaryk-Universität MUNI/41/006/2009 im Zeitraum von Mai bis August 2009 realisiert $^{1}$. Zur Zusammenarbeit am Projekt wurden sowohl LektorInnen als auch KursteilnehmerInnen diverser Firmenkurse eingeladen. Die meisten Kurse fanden bzw. finden in Brünn oder in der Umgebung statt. Beide Gruppen von ProbandInnen bekamen einen elektronischen Fragebogen per Email zugeschickt. Der Fragebogen für LektorInnen enthielt 17 Items, der für KursteilnehmerInnen 16 Items. Aus Platzgründen wird in diesem Artikel lediglich der erste Teil der Daten präsentiert, die sich aus den Fragebögen für LektorInenn ergaben. Der zweite Teil wird in der nächsten Nummer dieser Zeitschrift veröffentlicht.

\subsection{Der Fragebogen}

Der Fragebogen wurde an ca. 50 LektorInnen per E-Mail verschickt, von denen 22 ein komplett ausgefülltes Formular zurückschickten. Der Fragebogen hatte folgende drei Teile:

Teil I: Technisch-organisatorische Fragen über den Sprachkurs

Teil II: Methodik und Didaktik im Sprachkurs

Teil III: Berufsprofil des/der Lektors/Lektorin

Die meisten Items wurden als geschlossene bzw. halb-offene Fragen formuliert. Allerdings gab es auch offene Fragen, wenn z. B. eigene Erfahrungen oder Anregungen der LektorInnen bezüglich des Fremdsprachenunterrichts in Firmenkursen erfragt wurden.

\subsection{Datenauswertung und Ergebnisse}

Für die Auswertung der erfassten Daten wurde die Software Statistica 9 verwendet. Die Ergebnisse werden durch Säulen- bzw. Tortendiagramme oder Tabellen illustriert und beziehen sich nur auf die Stichprobe (22 ProbandInnen). Da die Stichprobe nicht genügend groß ist und dadurch die Prozentsätze verzerrend wirken könnten, fügen wir in den jeweiligen Graphiken neben den Prozentangaben noch die Anzahl der Antworten hinzu.

\section{Teil I: Technisch-organisatorische Fragen über den Sprachkurs}

Frage Nr. 1: Wie ist das Sprachniveau in Ihrem Kurslaut dem Gemeinsamen europäischen Referenzrahmen für Sprachen?

1 An der Durchführung der Untersuchung beteiligte sich außer der Autorin des Artikels auch Pavel Furch, Student an der Pädagogischen Fakultät der MU. Sein Beitrag bestand u. a. in der Datenorganisierung und -auswertung. 
Durch die erste Frage sollte festgestellt werden, auf welchem Sprachniveau sich die Kurse befinden, in denen die befragten LektorInnen unterrichten.

\section{Wie ist das Sprachniveau in Ihrem Kurs laut dem Gemeinsamen europäischen Referenzrahmen für Sprachen?}

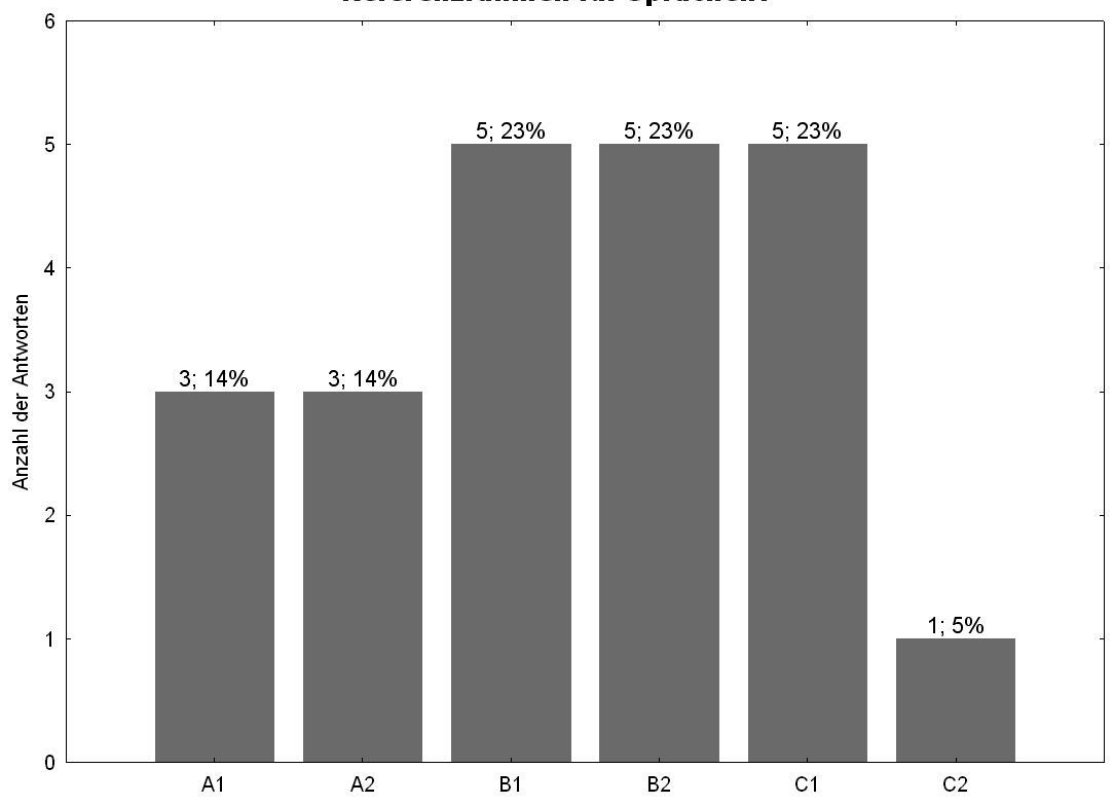

Auf den ersten Blick ist der Graphik zu entnehmen, dass die Firmenkurse auf allen Sprachniveaus verlaufen, wobei die Mehrheit der untersuchten Firmenkurse sich zwischen dem Niveau B1 und C1 befindet.

Frage Nr. 2: Wie wird der Sprachkurs abgeschlossen?

Die Art und Weise, wie der Sprachkurs abgeschlossen wird, gehört zu den oft diskutierten Themen der Fremdsprachendidaktik. In Bezug auf den Firmenunterricht stoßen wir auf mehrere Abschlussmöglichkeiten, die oft die Leitung der Firma selbst bestimmen will. Die folgende Graphik zeigt, wie die Kurse der befragten LektorInnen abgeschlossen werden. 


\section{Wie wird der Sprachkurs abgeschlossen?}

andere Form; 2; $9 \% \quad$ internationale Sprachprüfung; $1 ; 5 \%$

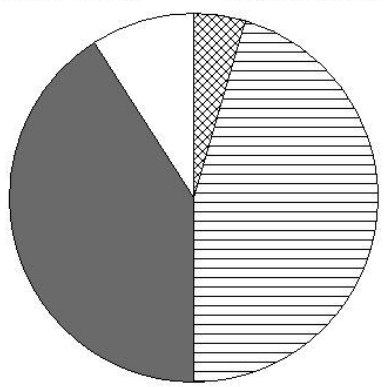

keine Anforderungen; $9 ; 41 \% \quad$ Abschlusstest; $10 ; 45 \%$

Aus der Umfrage geht hervor, dass fast die Hälfte der Sprachkurse mit einem schriftlichen Test abgeschlossen wird. Ein überraschend großer Teil der LektorInnen (41 \%) antwortete, dass es keine Anforderungen zum Abschluss seitens der Firmenleitung gebe. Eine andere Form des Abschlusses, die bei zwei Kursen angegeben wurde, bedeutet, dass dem Test noch eine Evaluation der abgegebenen Leistungen nach dem Gemeinsamen europäischen Referenzrahmen für Sprachen folgt oder dass man zwischen einem Test und einer internationalen Sprachprüfung wählen kann. Nur ein Kurs führt explizit zu einer internationalen Sprachprüfung.

Frage Nr. 3: Wer bestimmt den Inhalt des Sprachkurses?

Zum Thema „Inhalt des Sprachkurses“ gab es in dieser Untersuchung gleich zwei Fragen. Eine betrifft die Person bzw. Institution, die darüber entscheidet, was gelernt wird, die zweite fragt direkt danach, was gelernt wird (siehe Frage Nr. 5). 


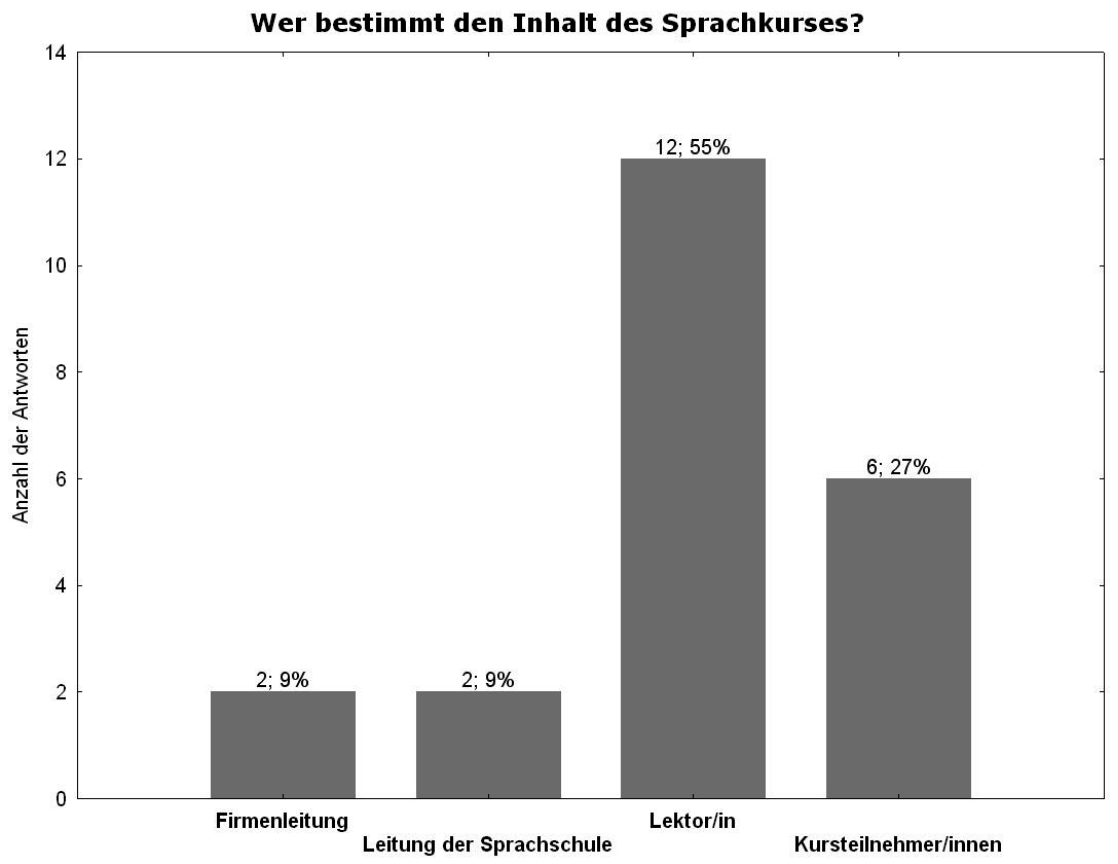

Von 22 befragten LektorInnen gaben 12 an, dass sie selbst den Unterrichtsinhalt festlegen. Beinahe in einem Drittel der Fälle wurde geantwortet, dass die KursteilnehmerInnen selbst an der Entscheidung über den Lernstoff teilnehmen. Diese Ergebnisse bestätigten auch unsere Prämisse, die besagte, dass die Inhalte nicht immer „von oben“ bestimmt werden. Jede Zielgruppe hat nämlich ganz andere Vorstellungen, was sie für den Beruf braucht und worauf sie unter Umständen verzichten kann. Aus meiner persönlichen Erfahrung würde ich empfehlen, die KursteilnehmerInnen über die Themenbereiche bzw. über das Lehrwerk mitbestimmen zu lassen. Dies erhöht nicht nur ihre Motivation, sondern auch die Effizienz des Kurses an sich.

Frage Nr. 4 Wie sind die Unterrichtsräume ausgestattet (Flipchart, Audio-Video, Kopiergerät, Internetanschluss etc.)?

Wenn der Sprachkurs direkt in der Firma stattfinden soll, muss zunächst geklärt werden, in welchem Raum der Unterricht verläuft. Meistens wird der Kurs in einem Konferenzraum oder in einem Büro abgehalten. Sehr selten wird für den Sprachunterricht ein extra Raum reserviert. Daher war das Ziel dieser Frage 
festzustellen, in wieweit diese „improvisierten Unterrichtsräume“ mit Medien bzw. anderen technischen Hilfsmitteln ausgestattet sind.

\section{Wie sind die Unterrichtsräume ausgestattet (Flipchart, Audio-Video, Kopiergerät, Internetanschluss etc.)?}

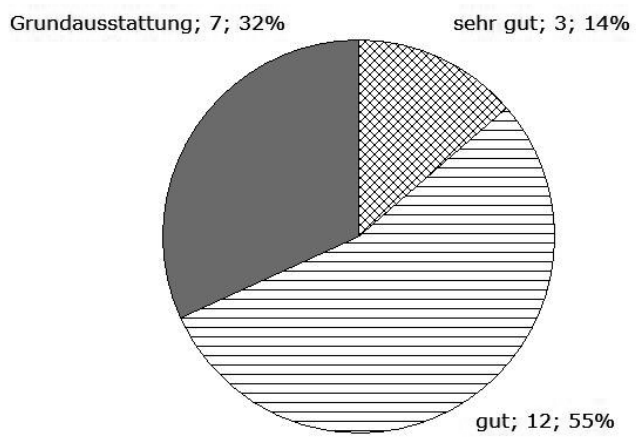

Zirka zwei Drittel der Befragten arbeiten in gut oder sehr gut ausgestatteten Räumen. Ungefähr ein Drittel der LektorInnen muss sich beim Unterrichten auf eine Grundausstattung beschränken und ggf. eigene Technik mitbringen.

\section{Teil II: Methodik und Didaktik im Sprachkurs}

Frage Nr. 5: Was stellt den Inhalt des Sprachkurses dar?

Mit dieser Frage wollte man feststellen, welche Sprachschicht im Unterricht bevorzugt wird. Es gab drei Möglichkeiten zur Auswahl:

a) nur Alltagssprache,

b) nur Fachsprache bzw. Berufssprache,

c) Kombination von Alltags- und Fachsprache. 


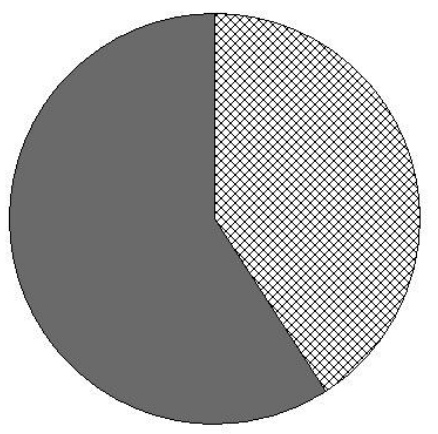

Kombination von Alltags- und Fachsprache; $13 ; 59 \%$

Da sich in diesem Fall nur zwei Gruppen von Antworten ergaben, ist eine graphische Darstellung überflüssig. Fast $60 \%$ der befragten LektorInnen antworteten, dass sowohl Alltagssprache als auch Fachsprache in ihren Kursen unterrichtet werden. Die erste Möglichkeit (nur Alltagssprache) gaben zirka 40 \% der ProbandInnen an. Die zweite Möglichkeit (nur Fachsprache) markierte keine/r.

Frage Nr. 6 Wie viel Aufmerksamkeit wird folgenden Tätigkeiten gewidmet?

Unter der Bezeichnung „Tätigkeiten“ war das Training einzelner Sprachfertigkeiten und Teilkompetenzen gemeint. Einfachheitshalber verzichteten wir jedoch im Fragebogen auf diese fachwissenschaftlichen Termini. Die ProbandInnen sollten jeder Fertigkeit bzw. Teilkompetenz eine Zahl auf einer Skala von 1 bis 5 zuordnen, je nachdem, ob sie sich der jeweiligen Tätigkeit sehr oft im Unterricht widmen (1) oder gar nicht widmen (5), wobei man die Eins höchstens zwei Mal verwenden durfte. Die Ergebnisse sind in der folgenden Tabelle nach dem Durchschnittswert geordnet.

wird sehr oft gewidmet 1-2-3-4-5 wird gar nicht gewidmet

\begin{tabular}{|c|l|c|}
\hline $\begin{array}{c}\text { Platz in der } \\
\text { Reihenfolge }\end{array}$ & \multicolumn{1}{|c|}{ Tätigkeit } & Durchschnittswert \\
\hline 1 & Konversation & 1,5 \\
\hline 2 & Wortschatz & 1,8 \\
\hline 3 & Grammatik & 2,1 \\
\hline 4 & Lesen & 2,2 \\
\hline
\end{tabular}




\begin{tabular}{|l|l|l|}
\hline 5 & Hören & 2,6 \\
\hline 6 & Aussprache & 3,3 \\
\hline 7 & Schreiben & 3,7 \\
\hline 8 & Rechtschreibung & 3,9 \\
\hline
\end{tabular}

An den Durchschnittswerten ist es abzulesen, dass Konversationsübungen und Wortschatzarbeit zu den häufigsten Aktivitäten in den untersuchten Firmenkursen gehören, Schreibaufgaben bzw. Rechtschreibübungen hingegen nicht so oft gemacht werden.

Frage Nr. 7 Welche Sozialform verwenden Sie oft im Firmenkurs?

Bei dieser Frage konnten die LektorInnen mehrere Antworten ankreuzen. Zur Auswahl bekamen Sie folgende Alternativen: Frontalunterricht, Gruppenarbeit, Partnerarbeit, Einzelarbeit oder andere Sozialform. Zu der letztgenannten Antwort konnte eine Beschreibung hinzugefügt werden. Diese Möglichkeit wurde jedoch von niemandem genutzt.

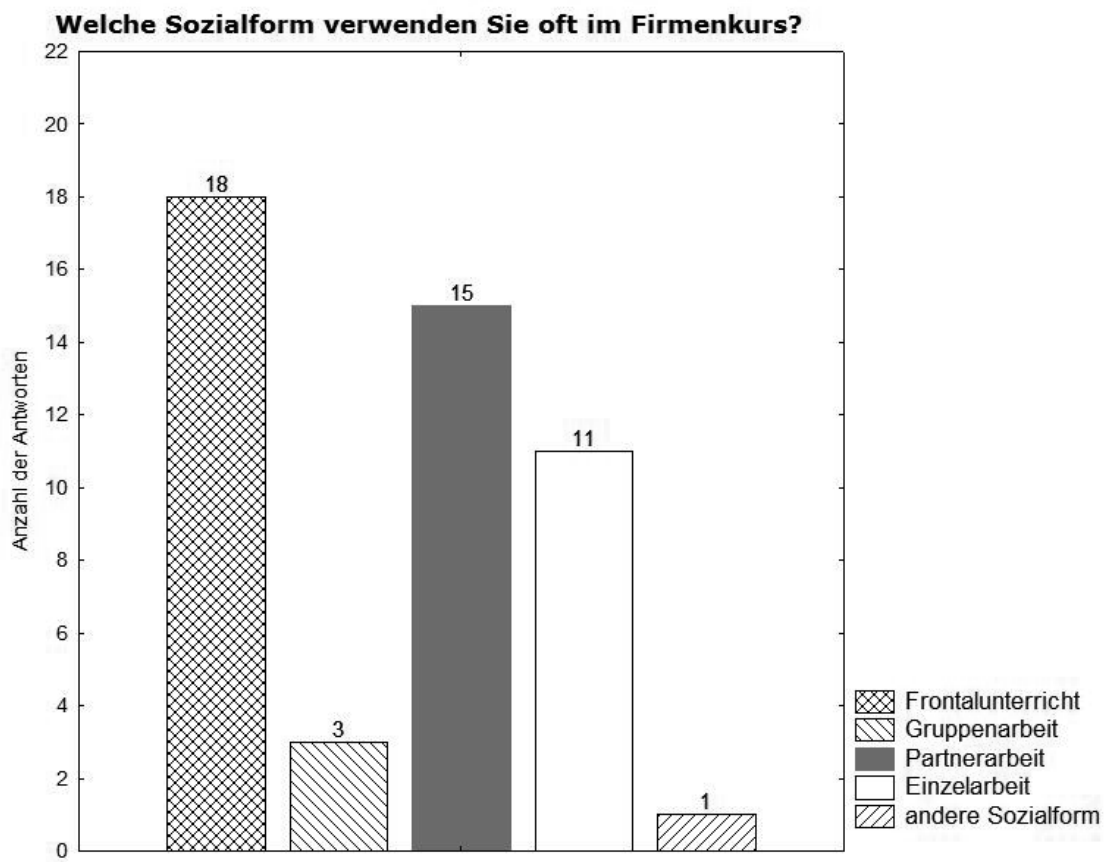


$\mathrm{Da}$ in diesem Fall mehrere Antworten markiert werden konnten, sind in der Graphik nur Zahlen ohne Prozentangaben angeführt, die zeigen, wie oft die jeweilige Sozialform angegeben wurde. Wie die Graphik illustriert, gab die Mehrheit der LektorInnen an, dass Sie oft frontal unterrichten. Die Partnerarbeit wurde insgesamt 15x markiert, was auch auf eine häufig eingesetzte Sozialform hindeuten kann. Die Einzelarbeit scheint von der Hälfte der Befragten verwendet zu werden. Im Gegensatz dazu wird nicht so oft in Gruppen gearbeitet. Der Grund dafür mag die oft niedrige Anzahl der KursteilnehmerInnen in solchen Sprachkursen sein.

Frage Nr. 8 Welche Methode bzw. Methoden des Fremdsprachenunterrichts halten Sie im Firmenkurs für besonders effektiv?

Die Frage der Unterrichtsmethoden wurde ganz offen gestellt, d. h. ohne Alternativantworten. Im Einklang mit den Ergebnissen von Frage Nr. 6 waren die am häufigsten erwähnten Methoden kommunikativ gerichtete Konversationsspiele, Rollenspiele und Dialoge. Oft werden solche Methoden verwendet, die den KursteilnehmerInnen ein individuelles Lerntempo ermöglichen. Diese verlaufen dann in der Form von Einzel- oder Partnerarbeit (Mind Mapping, Kreuzworträtsel, Wortspiele etc.). Nicht zuletzt wurden auch Drillübungen als Methode für die Grammatikwiederholung angegeben. In einem Fragebogen stand, dass auch Texte gelesen werden, über die dann diskutiert wird. Damit sind wir wieder bei der Konversation, die als bevorzugte Aktivität im Rahmen der untersuchten Kurse gelten kann.

Frage Nr. 9 Welche Methode des Fremdsprachenunterrichts kann im Firmenkurs gar oder nur unter großen Schwierigkeiten angewendet werden?

Ähnlich wie die vorhergehende Frage wurde auch diese Frage offen gestellt. Da die meisten Kurse, über die die befragten LektorInnen berichteten, nicht zahlreich besucht waren, beziehen sich viele Antworten auf die Methoden, die eine Gruppenarbeit voraussetzen wie z. B. Spiele, wo zwei Teams gegeneinander kämpfen, oder andere Spiele für mehrere SpielerInnen. Nach einer Aussage wäre es auch schwierig einen Projektunterricht zu planen und zu realisieren. Dies hängt nicht nur mit der Größe der Gruppe zusammen, sondern auch mit anderen Faktoren, die im Rahmen der nächsten Frage thematisiert werden. Im Falle von Individualunterricht ist natürlich nicht einmal Arbeit in Paaren möglich. In einem Fragebogen stand auch, dass man nicht lange frontal unterrichten kann oder lange mit dem Lehrwerk arbeiten soll. Der Unterricht sollte kreativ gestaltet werden. 
Frage Nr. 10 Was stört den Unterricht im Firmenkurs?

Bei dieser Frage wurden sowohl Alternativantworten als auch eine Möglichkeit, eigene Aussage zu formulieren, zur Verfügung gestellt. Man konnte wieder mehrere Antworten markieren, daher zeigt die folgende Säulengraphik nur die Anzahl, wie oft die jeweilige Antwort angegeben wurde, und keine Prozentsätze.

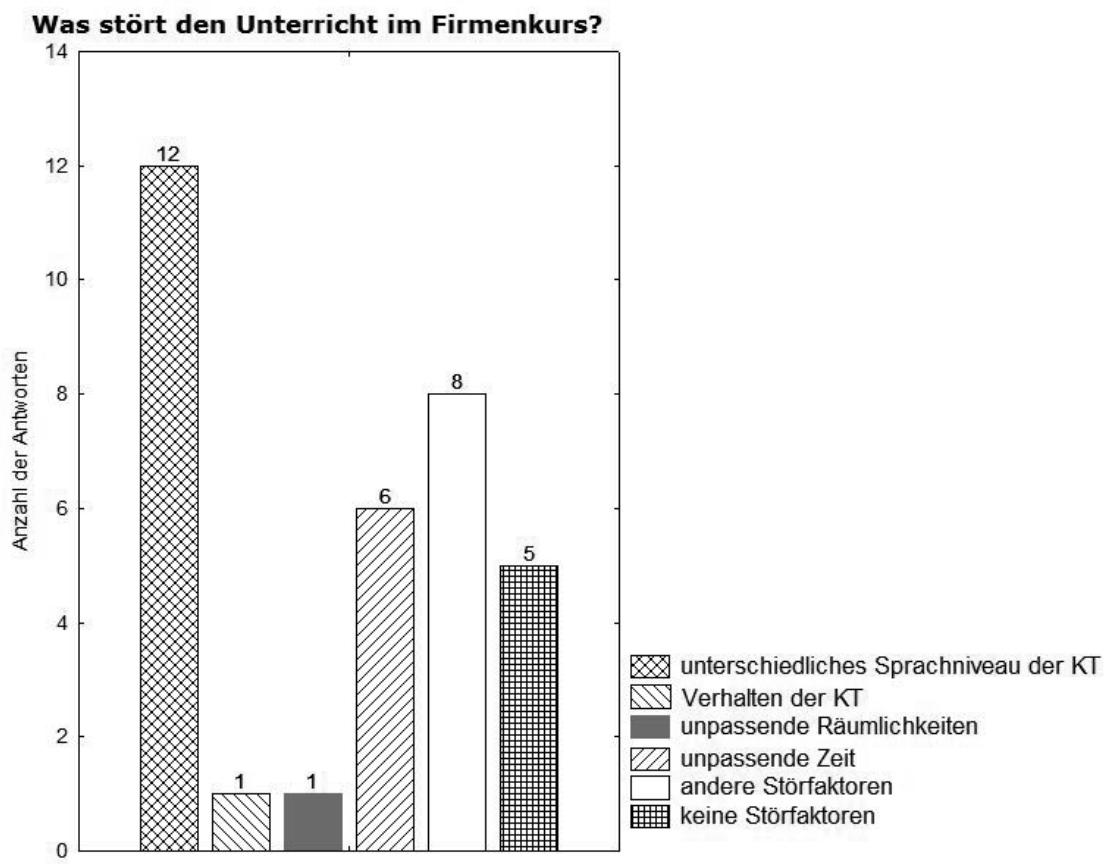

Im Falle der Auswahl unter verschiedenen Alternativantworten stellt den häufigsten Störfaktor beim Unterricht in Firmenkursen das unterschiedliche Sprachniveau der KursteilnehmerInnen dar. Darüber hinaus wurde sechsmal die Antwort unpassende Zeit für den Sprachkurs ausgewählt und je einmal Verhalten der KursteilnehmerInnen und unpassende Räumlichkeiten für den Unterricht. In acht Fragebögen stand, dass andere Störfaktoren vorkommen, die anschließend näher beschrieben wurden. Aus diesen frei formulierten Aussagen der befragten LektorInnen ergab sich, dass der Kurs vor allem durch eine unregelmäßige Präsenz der KursteilnehmerInnen gestört wird. Sehr oft kommen sie zu spät zum Unterricht oder werden sogar im Laufe des Unterrichts zum Arbeitsplatz gerufen. Auch kurze oder längere Dienstreisen und dadurch schwankende Zahl von KursteilnehmerInnen im Unterricht beeinflussen negativ die Lernprogression. Nicht zuletzt werden die KursteilnehmerInnen oft in 
der Arbeit überfordert und sind daher im Sprachkurs müde, wenig motiviert und haben keine Zeit und Lust zum Hausaufgabenmachen.

Laut fünf ProbandInnen wird der Unterricht durch nichts gestört.

Frage Nr. 11 Wie würden Sie die Gesamtatmosphäre im Firmenkurs bewerten?

Den befragten LektorInnen hatten fünf Möglichkeiten zur Auswahl: zwei positive (angenehm, eher angenehm), zwei negative (unangenehm, eher unangenehm) und eine neutrale Bewertung (neutral).

\section{Wie würden Sie die Gesamtatmosphäre im Firmenkurs bewerten?}

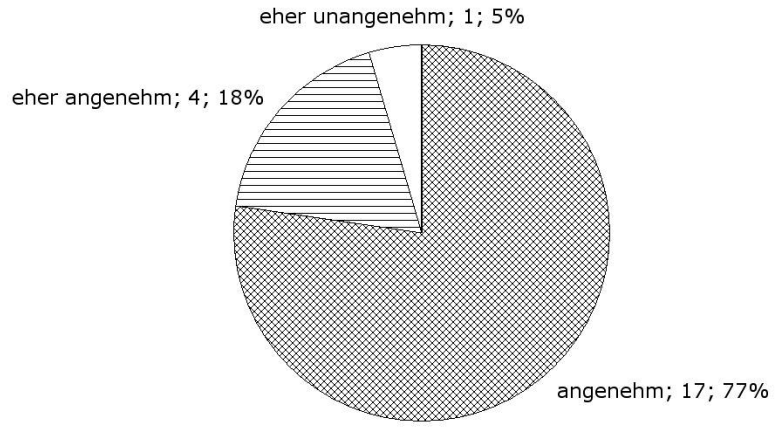

Die meisten LektorInnen bewerten die Atmosphäre in Ihrem Firmenkurs als angenehm bzw. eher angenehm. Nach einer Aussage ist die Atmosphäre eher unangenehm. Andere Antworten (unangenehm, neutral) blieben ungenutzt.

Frage Nr. 12 Wie wird Ihr Unterricht seitens der KursteilnehmerInnen meistens bewertet?

Bei der zwölften Frage gab es nur vier Alternativantworten (positiv, negativ, neutral oder ich habe kein Feedback). Obwohl die Antworten keine objektive Information über die Qualität des Unterrichts liefern können, zeigen sie doch eine gewisse Tendenz, die im Rahmen einer weiteren Untersuchung überprüft werden könnte. 


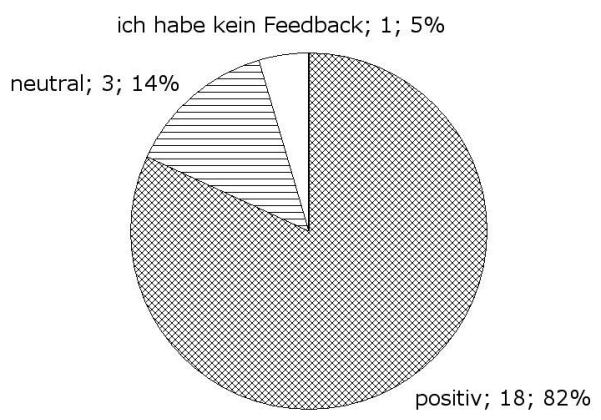

DiemeistenLektorInnenbehaupten, dassIhrUnterrichtvonden KursteilnehmerInnen positiv bewertet wird. Drei weitere ProbandInnen antworteten, dass Ihr Unterricht neutral bewertet wird. Ein/e Lektor/in hat angeblich keine Rückmeldungen. Keine/ r entschied sich für die letzte Möglichkeit (negativ).

\section{Teil III: Berufsprofil des/der Lektors/Lektorin}

Frage Nr. 13 Welchen Schulabschluss haben Sie?

Mit dieser Frage wollten wir feststellen, wie qualifiziert die LektorInnen in den Firmenkursen sind, die in Firmenkursen unterrichten. Drei Antworten standen zur Auswahl: Mittelschulabschluss, Hochschulabschluss mit pädagogischer Ausrichtung oder Hochschulabschluss ohne pädagogische Ausrichtung).

\section{Welchen Schulabschluss haben Sie?}

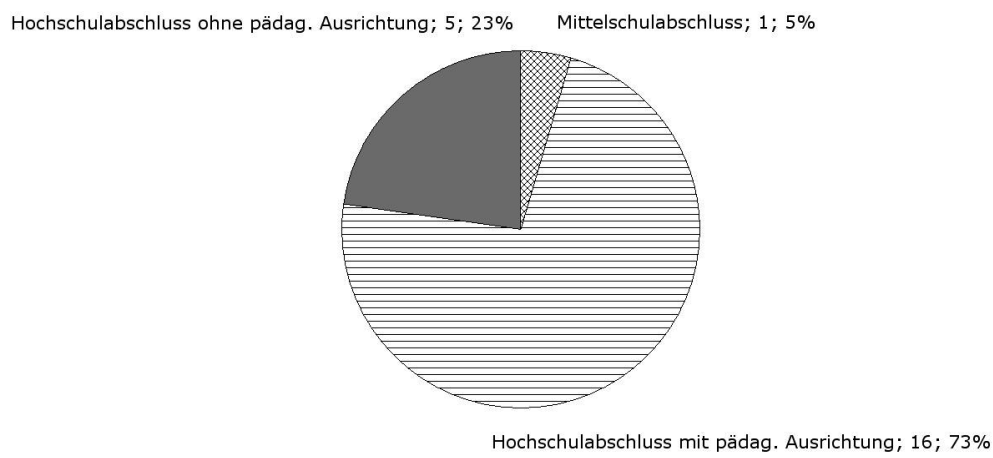


Es ist nicht besonders erfreulich zu erfahren, dass nur $73 \%$ der befragten LektorInnen über einen Hochschulabschluss in den Fächern Erziehungswissenschaft, Psychologie und Fachdidaktik verfügen. Hiermit bestätigt sich unsere weitere Hypothese, dass in Firmenkursen oft unqualifizierte LektorInnen unterrichten. In manchen Fällen sind es zwar ausgebildete LehrerInnen, die allerdings eine Ausbildung im Bereich der Sekundarstufe oder Gymnasien absolviert haben. In einem Firmenkurs stellen sie dann früher oder später fest, dass der Unterricht wesentlich verschieden von dem in einer Schule ist: andere Altersgruppe, andere Anforderungen seitens der Firmenleitung oder der KursteilnehmerInnen, andere technische und zeitliche Bedingungen etc. Daher wäre es sinnvoll eine neue Fachrichtung oder ein neues Aufbaustudium anzubieten, wo die Studierenden speziell für den Firmenunterricht ausgebildet und damit besser vorbereitet würden.

Item Nr. 14 wurde nicht als Frage sondern als Aussagesatz formuliert, der vollendet werden sollte und der sich auf das gegenwärtige Interesse der LektorInnen, sich auszubilden bzw. weiterzubilden, bezieht.

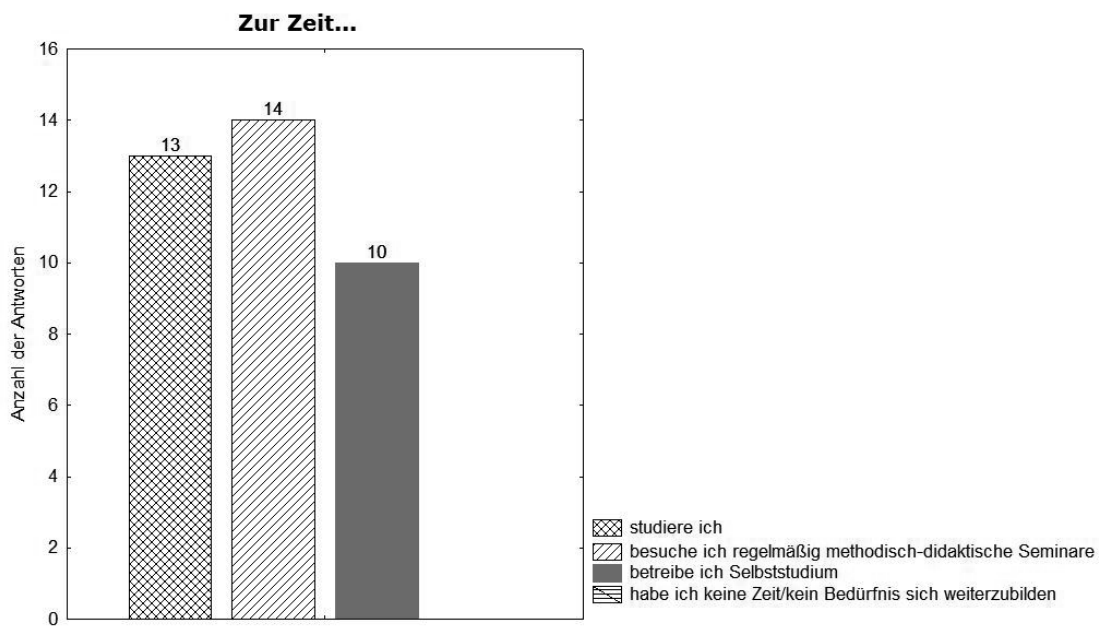

Ähnlich wie bei Frage Nr. 7 oder 10 konnte man sich auch hier für mehrere Möglichkeiten entscheiden. Die Ergebnisse zeigen, dass mehr als eine Hälfte der ProbandInnen regelmäßig methodisch-didaktische Seminare besucht. Zehnmal wurde angegeben, dass sie auch Selbststudien betreiben (Lesen von Fachlektüre etc.). Dreizehn LektorInnen studieren noch entweder an der Pädagogischen oder an der Philosophischen Fakultät. In einem Fall wurde die Fakultät für Sozialwissenschaften angegeben. 
Neben der methodisch-didaktischen Ausbildung wollten wir feststellen, in wieweit die LektorInnen die deutsche Sprache beherrschen. Dazu dienten Fragen nach Auslandsaufenthalten, Sprachprüfungen bzw. anderen Möglichkeiten, sich sprachlich zu vervollkommnen.

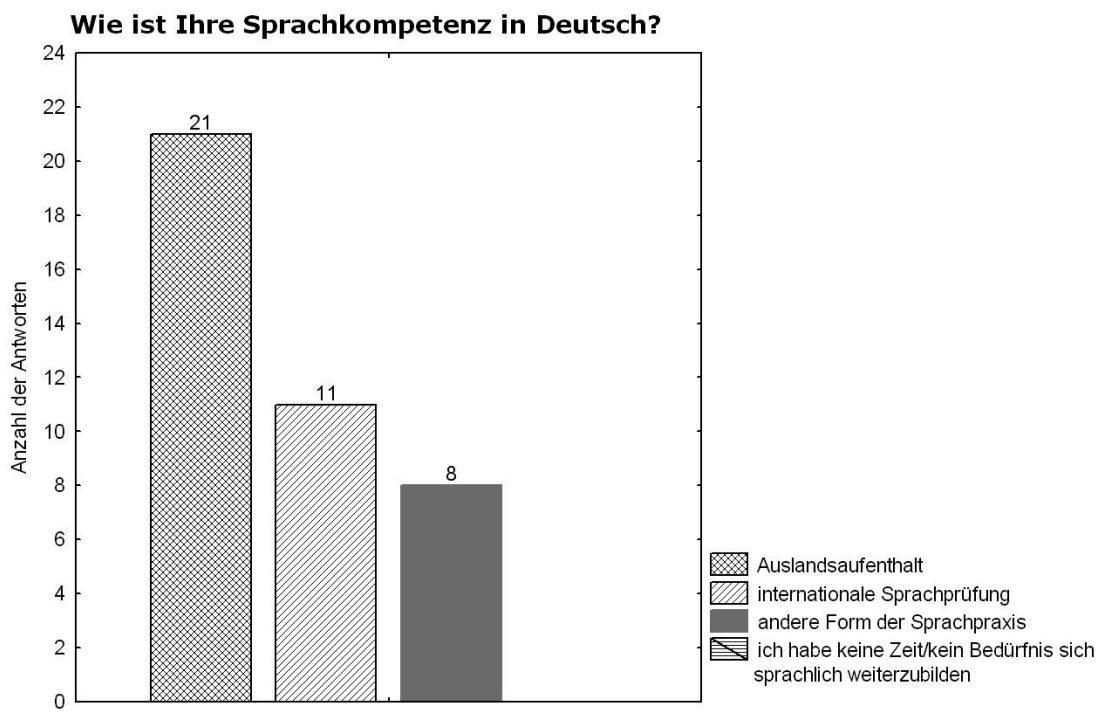

Es ist erfreulich, dass alle LektorInnen bis auf eine/n einen längeren Aufenthalt im Ausland absolvierten. Zu dieser Antwort sollten sie noch ergänzen, wie lange der Aufenthalt gedauert hat. Hier antworteten leider nur elf von ihnen: die kürzeste Aufenthaltsdauer betrug sieben Monate, die längste drei Jahre. Weiter kann man der Graphik entnehmen, dass genau die Hälfte der LektorInnen eine internationale Sprachprüfung bestanden hat. Diesmal sollte der Name der Prüfung ergänzt werden, was jedoch nur vier ProbandInnen taten. Daher darf man aus diesen Ergebnissen keine Schlüsse darüber ziehen, wie sprachkompetent die LektorInnen sind, wenn wir keine Vorstellung über das Niveau der Prüfungen haben. Acht LektorInnen vervollkommnen ihr Deutsch auf eine andere Art und Weise (z. B. durch Lektüre, Fernsehen, Übersetzungen und Dolmetschen, ständigen Kontakt mit der Sprache bzw. mit MuttersprachlerInnen, Internet). 
Frage Nr. 16 Wie lange unterrichten Sie schon?

Die nächste Frage bezog sich auf die pädagogischen Erfahrungen der LektorInnen. Sie sollten angeben, wie lange sie schon unterrichten, indem sie eine der fünf Antworten ankreuzten: weniger als $1 \mathrm{Jahr}, 1-3 \mathrm{Jahre,} \mathrm{3-5} \mathrm{Jahre,} \mathrm{5-10} \mathrm{Jahre,} \mathrm{mehr}$ als 10 Jahre.

\section{Wie lange unterrichten Sie schon?}

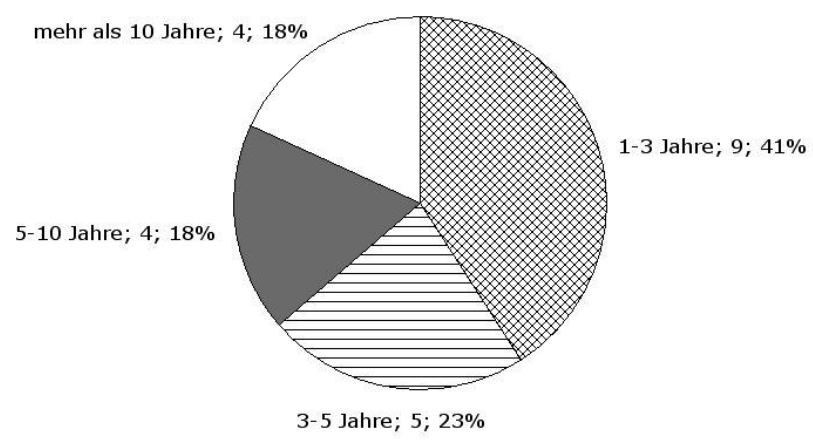

Zusammenfassend kann man sagen, dass $64 \%$ der Befragten länger als ein Jahr aber kürzer als fünf Jahre unterrichten. Bezüglich der Stichprobe kann man schlussfolgern, dass in den Firmenkursen eher jüngere LektorInnen - vielleicht frische HochschulabsolventInnen - unterrichten. Diese Hypothese wäre allerdings noch mit einem weiteren Fragebogen, der demographisch ausgerichtet wäre, zu überprüfen.

Am Ende des Fragebogens wurden die LektorInnen aufgefordert, weitere Anregungen bzw. Tipps bezüglich der Effizienz des Firmenunterrichts aufzuschreiben. An den Aussagen ist abzulesen, dass die größte Aufgabe des/der Lektors/in darin besteht, die KursteilnehmerInnen zu motivieren. Es wurde betont, dass es nicht reicht, wenn sie den Kurs bloß besuchen, um „die Sprache nicht zu vergessen“. Es ist viel besser, wenn ein konkretes Ziel formuliert wird. Es muss nicht immer eine Sprachprüfung sein, sondern klare Zielsetzungen, die jede/r Kursteilnehmer/in am Anfang des Kurses individuell festlegt bzw. anderen KursteilnehmerInnen präsentiert. Am Ende des Kurses sollte dann eine Evaluation folgen, um bewusst zu machen, was erreicht wurde und was nicht.

Motivieren sollte man auch durch einen abwechslungsreichen Unterricht, denn viele KursteilnehmerInnen kommen erschöpft von der Arbeit und haben oft keine Lust mitzumachen. Daher müssen die Aktivitäten gut methodisch-didaktisch, 
aber auch psychologisch durchdacht werden, damit sie die Zielgruppe wirklich ansprechen und „aufwecken“.

Aus Platzgründen wurden die Ergebnisse zu jeder Frage zusammenfassend präsentiert bzw. kurz interpretiert. Allerdings steht potenziellen Interessenten die vollständige Datenbank bei der Autorin des Artikels zur Verfügung. ${ }^{1}$

\subsection{Kritische Reflexion und Ausblick}

Diese Umfrage ist als Voruntersuchung im Bereich des Deutschunterrichts in Firmenkursen gedacht, die zu einer groben Deskription der gegenwärtigen Situation dienen sollte. Obwohl die Ergebnisse sich nicht verallgemeinern lassen, weil die Stichprobe nicht ausreichend groß war, ergaben sich im Laufe der Untersuchung weitere Fragen, die detaillierter erforscht werden sollten. Im Prinzip könnte man jeden Teil des vorhandenen Fragebogens als einen selbständigen Fragebogen verwenden und diesen um weitere, tiefer eingehende Fragen zu bereichern. Es zeigte sich, dass die offen formulierten Fragen von großer Bedeutung sind. Hier sammeln sich nämlich wertvolle Informationen, die im Voraus nur schwer abzuschätzen gewesen wären.

Zusammenfassend kann man sagen, dass der Sprachunterricht in Firmenkursen einen spezifischen Zugang verlangt. Als Lektor/in sollte man damit rechnen, dass die Arbeit mit berufstätigen Lernern wesentlich unterschiedlich ist, als mit SchülerInnen oder StudentInnen. Dass vor allem Flexibilität und Improvisierungsfähigkeit erfordert werden, wenn ab und zu die Stunde wegen einer niedrigen Anzahl der KursteilnehmerInnen nicht nach dem geplanten Programm verlaufen kann. Dass die Motivation der Lernenden sowie Fleiß und Einsatz manchmal hinken, weil sie zu erschöpft von der Arbeit sind. Das ist pure Realität, auf die man sich allerdings vorbereiten könnte und zu diesem Zweck wäre es sinnvoll, wie schon oben gesagt wurde, ein spezialisiertes Aufbaustudium mit dem Schwerpunkt Fremdsprachenunterricht in Firmenkursen oder in anderen berufsorientierten Sprachkursen anzubieten.

\section{Literatur:}

NEKULA (2004): Nekula, Marek. Deutsch als Europasprache aus tschechischer Sicht, in: Lohse, Ch. (Hg.): Die deutsche Sprache in der Europäischen Union. Rolle und Chancen aus rechts- und sprachwissenschaftlicher Sicht. Baden-Baden: Nomos Verlagsgesellschaft 2004, $129-144$.

GERR (2000): Gemeinsamer europäischer Referenzrahmen für Sprachen: lernen, lehren und beurteilen (online). Europarat der EU, 2000. Abgerufen am 10. 9. 2009 unter: http://www.goethe.de/referenzrahmen.

1 Die Autorin ist unter der E-Mail-Adresse pmareckova@ped.muni.cz erreichbar. 